\title{
Treated with Hymen Sparing Surgery in Familial Imperforate Hymen
}

\author{
Mustafa Kaplanoglu
}

\section{ABSTRACT}

Imperforate hymen is a rare congenital female genital tract obstructive pathology. Although it is generally sporadic and isolated, very rare familial occurrence cases have been published in the literature. We present a rare case of the familial occurrence of IH and its surgical treatment. This case was admitted to our clinic with chronic pelvic pain, difficulty urination and pelvic mass. After the gynecologic examination and ultrasound view, the diagnosis of IH was made. Her sister who two years older than she had been previously evaluated for amenorrhea and her sister had undergone surgical procedures for IH. Because of Hymen is an important symbol of virginity in family culture, hymen sparing surgery was performed. The familial occurrence of IH is a rare condition and very few reported cases in the literature. Hymenectomy can cause social retraction in cases therefore hymen sparing surgery is an important surgery, especially Muslim cultures.

Keywords: Familial occurence, imperforate hymen, surgery.

Submitted: September 3, 2021

Published: September 28, 2021

ISSN: 2593-8339

DOI: $10.24018 /$ ejmed.2021.3.5.1040

\section{Kaplanoglu*}

Department of Obstetric of Gynecology, Health Sciences University, Adana City Training and Research Hospital, Adana, Turkey. (e-mail:

mustafakaplanoglu@gmail.com)

*Corresponding Author

\section{INTRODUCTION}

Imperforate hymen (IH) is an uncommon congenital gynecologic condition, with an approximately incidence of 1 in 1,000 females. The hymen completely obstructs the vaginal opening. It can be confused with vaginal agenesis, transverse vaginal septum, OHVIRA syndrome (Herlyn Werner Wunderlich Syndrome=obstructed hemi-vagina and ipsilateral renal anomaly) or RKHM syndrome (RokitanskyKuster-Hauser-Mayer Syndrome) which are congenital obstructive pathologies [1].

Most of cases are diagnosis at puberty. The most common complaints of $\mathrm{IH}$ are amenorrhea, chronic/acute pelvic pain, urine retention, constipation. IH is generally a sporadic pathology but it can be the rarely familial occurrence. The specific genetic mutation has not been defined. Very few familial IH cases have been published in the literature. The standard treatment of IH is surgical hymenectomy (annular, X shape...) [2].

In the present study, hymen sparing surgery and familial occurrence has presented in this case

\section{CASE REPORT}

In this presented case, a 13 years-old girl was admitted to the adolescent gynecology department with a last one-year pelvic pain, difficulty urination and pelvic mass. On pelvic gynecologic examination, there was a bulging bluish membrane, hymen was closed and bulging outward (Fig. 1).

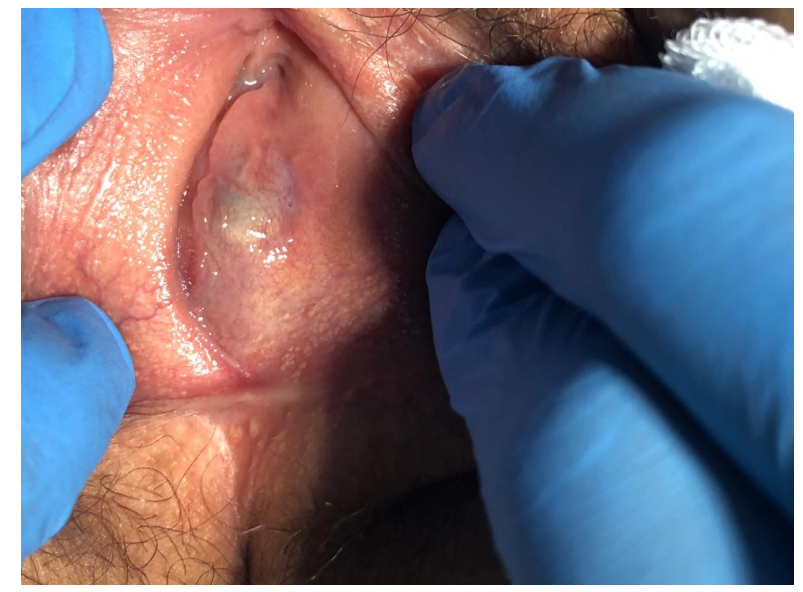

Fig. 1.Imperforate hymen view before surgery.

On the rectal examination, the rectum was compressing by mobile central pelvic mass. Secondary sexual characteristics were present (pubic/axillary hair and breast buds = Tanner III). Abdominopelvic ultrasound at the gynecology clinic was seen ovoid mid-pelvic sonolucent (like endometrioma) pelvic mass extending from the lower vagina to the level umbilicus. The uterus was seen on this mass. The mass sizes were $18 \times 8 \times 8.5 \mathrm{~cm}$ and filled with dense liquid. The mass was evaluated as hematocolpos and hematometra. The bladder was distended. Adnexal pathology was no detected. According to the findings, IH was thought, and the patient was prepared to surgical intervention. Also, we learned that her older sister who two years older than she, had been previously evaluated for amenorrhea and her sister had undergone surgical 
procedures for IH. After the patient informed consent form was signed. Int he surgery, the surgical line of the hymen was sutured (marked) at the level of 3, 6, 9, 12 levels with no 3-0 vicryl for annular hymenectomy (Fig. 2). Afterward, annular hymenectomy was performed. Approximately 2000 cc dark, old blood, giving it a chocolate like appearance fluid was drained. The incision line was sutured with no 3-0 vicryl. Vagen was irrigated with saline and the operation was finished (Fig. 3). There was no detected any complaint and complication in postoperative follow-up. The incision line healed without a problem.

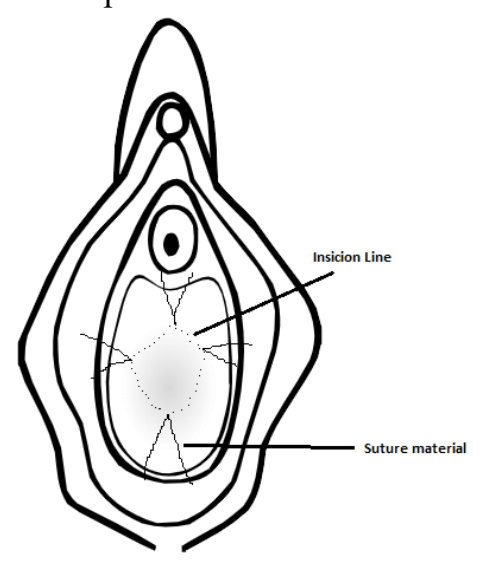

Fig. 2. Schematic view of the annular hymenectomy.

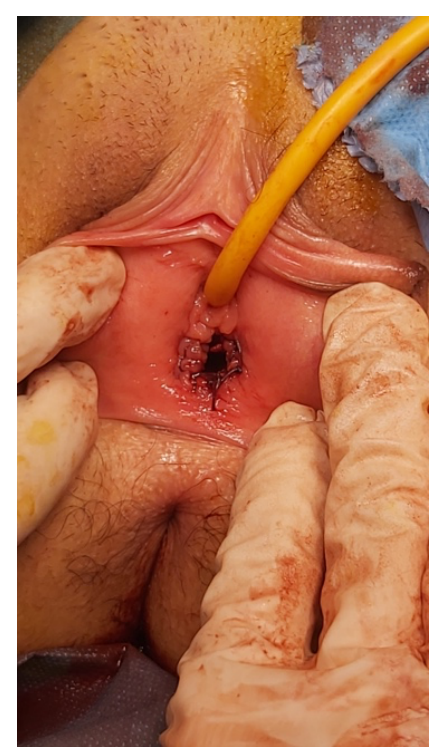

Fig. 3. External view of the imperforate hymen after surgery.

\section{DISCUSSION}

$\mathrm{IH}$ is not a frequent condition, but it is the most common obstructive congenital abnormality of the female genital tract. This pathology is generally an isolated anomaly. But it is rarely associated with other anomalies such as vaginal/uterine septum, partial atresia of the vagina, duplicated uterus), anorectal and vertebral abnormalities and can be familial (autosomal dominant and recessive) [3]. But the specific gene location has not detected. This condition is usually no diagnosed until she complained of the primer amenorrhea. The clinical presentation of these cases is mostly lower abdominal mass, primer amenorrhea, urinary retention, constipation, acute abdomen. The cause of this complains are the accumulation of blood that it can be mass in the vagina (hematocolpos) and uterus (hematometra) after menarche. Vaginal (Hydrocolpos) and uterine (Hydrometra) cavity can be filled by secretion in the prepubertal period and similar symptoms can be seen [4]. On the other hand, the incidence of prenatal diagnosis is $6.7 \%$ of all $\mathrm{IH}$ cases $[5]$.

The diagnosis of IH is easy and is different in women's life periods. Firstly, hydrocolpos and/or hydrometra due to secretion can be detected in the late third trimester evaluation. In later periods of life (until puberty), it can be seen with a simple vulvar examination by mother or doctor. In puberty, classical symptoms are seen.

The suspicion must be necessary for the diagnosis especially in the prepubertal period. After the suspicion, the vulvar examination is enough. Ultrasonography/MRI are helpful in the diagnosis of complications and other abnormalities

If the diagnosis is late, several complications can be seen such as pelvic infection, hydronephrosis.

The treatment of this pathology is surgical. Several surgical techniques are defined (X shape incision, annular...). The purpose of the surgery is creating a permanent communication between the uterine cavity and vestibulum. The hymen is an important for virginity in some cultures (especially Muslim culture). Therefore, hymen saving surgery is important for avoid social problems. We are performed an annular hymenectomy operation in these cases, so the surgery is no social problem [6].

Medical and familial history is important for IH cases (Pain nature, the time of starting the complaints, mother, and sister anamnesis) An addition, endometriosis is an important gynecologic condition in reproductive age women. Retrograde menstruation is a causative theory in this pathology. Although $\mathrm{IH}$ is a generally benign condition, it can be led to retrograde menstrual flow. Therefore, IH can be a causative factor for endometriosis [5].

Hymenetomy is a standard surgical technique for IH. The most common hymenetomy technique is $\mathrm{X}$ shape incision. But hymen is an important symbol for virginity in some cultures. Annular hymenetomy which is hymen saving surgical procedure can be the most suitable surgical procedure.

\section{CONCLUSION}

$\mathrm{IH}$ is a rarely gynecologic condition and correct diagnosis is an important for several complications such as pelvic infection, endometriosis. Although it is a generally sporadic gynecologic pathology, it can be seen familial. Susceptible genes cannot be detected. After the diagnosis, familial history and cultural characteristics must be evaluated for surgical treatment. The detailed menstrual history of the other female family members must be evaluated for the familial occurrence of $\mathrm{IH}$.

\section{REFERENCES}

[1] A. S. Eksioglu, H. A. Maden, G. Cinar and Y. Tasci Yildiz, "Imperforate hymen causing bilateral hydroureteronephrosis in an infant with bicornuate uterus," Case Rep Urol. 2012;2012:102683. doi: $10.1155 / 2012 / 102683$ 
[2] R. Sakalkale and U. Samarakkody, "Familial occurrence of imperforate hymen," J. Pediatr. Adolesc. Gynecol. 2005, 18, 427429.DOI: 10.1016/j.jpag.2005.06.008

[3] K. Bonello, Y. Tlili, O. Ben Zeid, H. Figoni and M. Mazeghrane, "Haematocolpos due to imperforate hymen disguised as constipation," J Paediatr Child Health. 2020;10.1111/jpc.14931. doi:10.1111/jpc. 14931

[4] A. P. Singh, A. K. Gupta, R. Pardeshi and G. Raipuria, "Hydrocolpos Caused by Imperforate Hymen in a Preterm Newborn," Saudi J Med Med Sci. 2019;7(2):124-125 doi: 10.4103/sjmms.sjmms_69_18

[5] K. H. Lee, J. Hong, H. J. Jung, H. K. Jeong, S. J. Moon, W. H. Parkand J. I. Shin, "Imperforate Hymen: A Comprehensive Systematic Review," J Clin Med. 2019; 7; 8: E56. https://doi.org/10.3390/jcm8010056

[6] C. Cetin, C. Soysal, G. Khatib, I. F. Urunsak, and T. Cetin, “Annular hymenotomy for imperforate hymen,” J. Obstet. Gynaecol. Res. 2016, 42, 1013-1015 https://doi.org/10.1111/jog.13010 